Article

\title{
Max-Min Rate Fairness Optimization for In-Band Full-Duplex IoT Networks: User Grouping and Power Control
}

\author{
Ngo Tan Vu Khanh ${ }^{1}$ (D) \\ 1 Department of Ecommerce Technology, School of Business Information Technology, University of Economics \\ Ho Chi Minh City, Ho Chi Minh City 70000, Vietnam; khanhntv@ueh.edu.vn
}

\begin{abstract}
The skyrocketing growth in the number of Internet of Things (IoT) devices will certainly pose a huge traffic demand for fifth-generation (5G) wireless networks and beyond. In-band full-duplex (IBFD), which is theoretically expected to double the spectral efficiency of a half-duplex (HD) wireless channel and to connect more devices, has been considered as a promising technology to accelerate the development of IoT. To exploit the full potential of IBFD, the key challenge is how to handle network interference (including self-interference, co-channel interference and multiuser interference) more effectively. In this paper, we propose a simple yet efficient user grouping method, where a base station (BS) serves strong downlink users and weak uplink users and vice versa in different frequency bands, mitigating severe network interference. We aim to maximize a minimum rate among all users subject to bandwidth and power constraints, which is formulated as a highly nonconvex optimization problem. By leveraging inner approximation framework, we develop a very efficient iterative algorithm to solve this problem, which guarantees at least a local optimal solution. Numerical results are provided to show not only the benefit of using full-duplex raido at BS, but also the advantage of the proposed user grouping method.
\end{abstract}

Keywords: In-band full-duplex radios; full-duplex self-interference; user grouping; user fairness; Internet of Things; nonconvex programming; transmit beamforming

\section{Introduction}

By 2020 it is estimated that the number of Internet of Things (IoT) devices will be 20.4 billion with a connection density of 1 million devices per $\mathrm{km} 2$ [1]. In addition, the global mobile data traffic is projected to reach 49 exabytes per month in 2021 [2], in which the IoT related data is the main driving force. These numbers are tremendous and will further increase over the coming years. It is clear that the successful deployment of IoT will enable many crucial applications, including Financial Technology (FinTech) services, automation factory and remote surgery, to name a few. To meet the aforementioned demands and accelerate the roll-out of the IoT, the industry and academic communities are currently investigating promising physical layer technologies for fifth-generation (5G) wireless networks and beyond, including multiple access techniques and in-band full-duplex (IBFD) communications [3-5].

IBFD communication that allows the simultaneous downlink transmission and uplink reception on the frequency band is expected to double the spectral efficiency compared to haft-duplex counterparts (i.e., frequency division multiple access and time division multiple access) [5,6]. To achieve this at the system level, self-interference (SI) caused by signal leakage from the transmit antennas to receive ones at a base station (BS) must be suppressed to a very low value, e.g. approximately equal to the background noise. Recently, advances in hardware design for IBFD communication have enabled the cost-effective implementation of the IBFD-based BS, where SI can be canceled down to a few $\mathrm{dB}$ above the background noise [7-11]. Although IBFD communication has been extensively considered in the literature, it is mainly focused on short-range communications due to its current limitations on SI suppression. Consequently, it is not too far-fetched to envisage a hyper-dense small cell deployment 
in beyond 5G, which will result in severe network interference, especially co-channel interference (CCI) caused by signals from uplink users to a downlink user using the same time-frequency resources [12-15]. In a nutshell, IBFD communication poses a number of technological challenges but exciting areas of endeavour that ought to be addressed to fully capitalize on its benefits.

\subsection{Related Works}

Due to imperfect SI suppression, IBFD communication has been considered primarily for small-cell scenarios. In particular, Nguyen et al. [12] investigated a joint optimization of downlink beamformer and uplink power allocation to maximize the spectral efficiency under power constraints of both BS and users. The formulated problem is nonconvex, and hence the iterative algorithms based on the determinant maximization and sequential parametric convex approximation methods were proposed for its solutions. To further improve the system performance, the authors in [16] proposed a joint design of the selection of BS's half-array antenna modes and user assignments which serves users in two separate time slots, managing the whole network interference more effectively. The application of IBFD to wireless-powered communication system was considered in $[17,18]$, which provides simultaneous wireless information and power transfer to users. In addition, the spectral efficiency maximization (SEM) for IBFD in multi-cell networks was considered in [15] with the perfect channel state information (CSI) and in [19] with the imperfect CSI (i.e., the worst-case robust design). It should be noted that the coordinated multi-point transmission in multi-cell networks can no longer provide high data rate for edge users due to strong inter-cell interference, while a joint coordinated multi-point transmission may require huge amount of backhaul signaling to be exchanged among BSs. More importantly, the common downlink transmission design in the aforementioned works is linear beamforming which causes high computation complexity due to the large size of the optimization variables. Thus, an efficient beamforming design with a low complexity for IBFD-based systems is required.

To mitigate the SI and CCI, user grouping has recently been considered for IBFD-based systems. The user grouping and time allocation were jointly designed in [14], where one communication time block is divided into separate mini-slots and each user is allowed to transmit its signal in multiple mini-slots to improve the overall throughput. Although this design is capable of canceling the harmful effects of both SI and CCI, it comes at the cost of high complexity since the number of beamforming vectors at each user is proportional to the number of groups/mini-slots. A simple user grouping method was proposed in [20] for IBFD-based non-orthogonal multiple access and in [21] for FD-assisted physical layer security. The idea of this user grouping method is to divide the whole cell into two regions (near and far regions) with respect to the distance from the BS. However, this method tends to provide poor performance if two groups of user are not well separated or all users are placed in the same region.

\subsection{Main Contributions}

To address the shortcomings mentioned above, in this paper we propose an efficient user grouping method based on the mean-square of the channel gain to effectively divide users into two disjoint groups in IBFD IoT networks. In addition, we also derive a low-complexity beanforning design based on zero-forcing (ZF) technique, which is suitable for networks of large size. To that end, we formulate a novel optimization problem to maximize the minimum (max-min) data rate among all users, guaranteeing user fairness. The optimization problem of interest is a highly non-convex programming, where the existing convex solvers are not capable of solving it directly. The global optimal solution may be obtained using Brute-Force Search method, but it comes at the cost of extremely high complexity. Towards practical applications, we develop novel transformations to convert the original nonconvex problem into a simple convex one and solve it efficiently by standard solvers. Our main contributions are summarized as follows:

- Aiming at max-min rate fairness, we propose a new user grouping method to divide all users into two groups which are served in different frequency bands. The proposed method helps not 
only mitigate network interference but also better exploit the spatial degrees-of-freedom (DoF) because the number of users served at the same time is significantly reduced. To reduce the complexity caused by the downlink beamforming, which has been widely done by the previous works, we develop a ZF beamforming that requires to solve the problem of scalar variables, instead of vectors.

- To solve the nonconvex problem, we resort to the inner approximation (IA) framework [22,23] to approximate nonconvex parts. We then develop an iterative algorithm for its solution which requires to solve a simple convex program at each iteration. The computational complexity is also provided and discussed.

- Numerical results are presented to demonstrate the effectiveness of the proposed method in terms of convergence speed and total data rate. The performance improvement of the proposed scheme over state-of-the-art approaches, i.e., half-duplex, conventional IBFD (without user grouping), and non-orthogonal multiple access (NOMA)-based schemes.

\subsection{Paper Organization and Notation}

The rest of the paper is organized as follows. Section 2 provides the system model and problem formulation for IBFD-based networks. The proposed algorithm using IA method is presented in Section 3. Numerical results and discussions are given in Section 4, while Section 5 concludes the paper.

Notation: We use $\mathbf{X}^{T}, \mathbf{X}^{H}$ and $\operatorname{tr}(\mathbf{X})$ to denote the transpose, Hermitian transpose and trace of a matrix $\mathbf{X}$, respectively. $\|\cdot\|$ and $|\cdot|$ indicate the Euclidean norm of a vector and the absolute value of a complex scalar, respectively. $\Re\{\cdot\}$ returns the real part of an argument. $x \sim \mathcal{C N}\left(0, \sigma^{2}\right)$ implies that $x$ is a circularly symmetric complex Gaussian random variable with zero mean and variance $\sigma^{2}$.

\section{System Model and Problem Formulation}

\subsection{Channel Model}

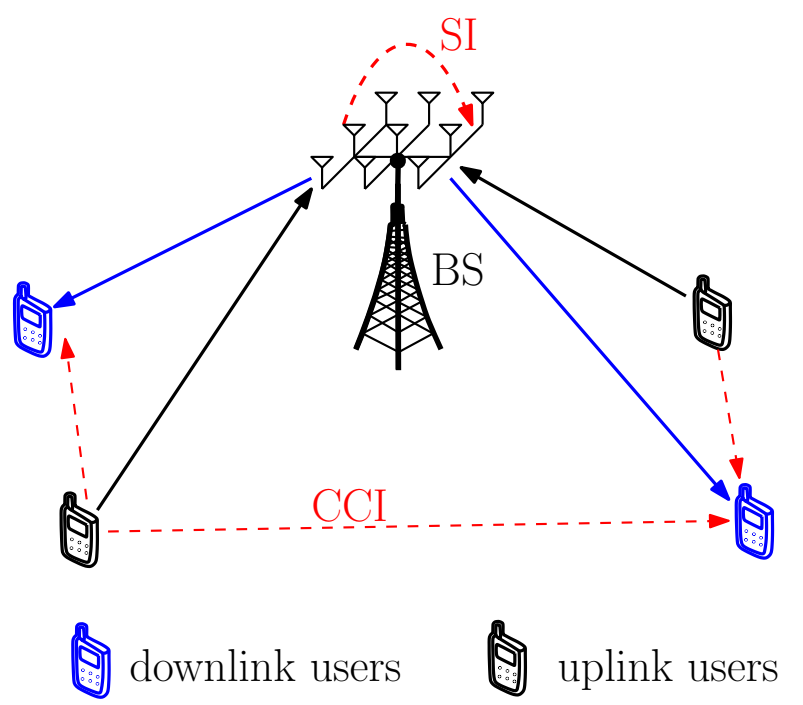

Figure 1. Illustration of IBFD-based systems wih multiple HD downlink users and uplink users.

We consider an IBFD communication system, where a BS is equipped with $N>1$ antennas to serve sets $\mathcal{D} \triangleq\{1,2, \cdots, D\}$ of $D=|\mathcal{D}|$ single-antenna downlink users and $\mathcal{U} \triangleq\{1,2, \cdots, U\}$ of $\mathcal{U}=|\mathcal{U}|$ single-antenna uplink users, as illustrated in Fig. 1. BS is equipped with IBFD capability using the circulator-based FD radio prototypes [7] to serve a group of haft-duplex downlink and uplink users in the same time-frequency resource. The channel vectors from BS to the $i$-th downlink user and from 
the $j$-th uplink user to BS are denoted by $\mathbf{h}_{i}^{\mathrm{dl}} \in \mathbb{C}^{1 \times N}, \forall i \in \mathcal{D}$ and $\mathbf{h}_{j}^{\mathrm{ul}} \in \mathbb{C}^{N \times 1}, \forall j \in \mathcal{U}$, respectively. The channel vectors $\mathbf{h}_{i}^{\mathrm{dl}}$ and $\mathbf{h}_{j}^{\mathrm{ul}}$ can be modeled as

$$
\mathbf{h}_{i}^{\mathrm{d} \mathrm{l}}=\sqrt{\varphi_{i}^{\mathrm{d}} \mathrm{h}} \overline{\mathbf{h}}_{i}^{\mathrm{dl}}, \forall i \in \mathcal{D}, \text { and } \mathbf{h}_{j}^{\mathrm{ul}}=\sqrt{\varphi_{j}^{\mathrm{ul}}} \overline{\mathbf{h}}_{j}^{\mathrm{ul}}, \forall j \in \mathcal{U}
$$

respectively, where $\varphi_{i}^{\mathrm{dl}}$ and $\varphi_{j}^{\mathrm{ul}}$ are the large-scale fading (e.g., path loss and shadowing), and $\overline{\mathbf{h}}_{i}^{\mathrm{dl}} \sim \mathcal{C N}\left(0, \mathbf{I}_{N}\right)$ and $\overline{\mathbf{h}}_{j}^{\mathrm{ul}} \sim \mathcal{C N}\left(0, \mathbf{I}_{N}\right)$ are the small-scale fading. For the imperfect SI suppression at BS, we assume that there still exists residual SI level $\rho \in[0,1)$ after all analog and digital cancellations. The lower the level of $\rho$ the lower the SI will cause to the uplink reception. The SI channel matrix between transmit and receive antennas is $\mathbf{H}_{\mathrm{SI}} \in \mathbb{C}^{N \times N}$, which is modeled as independent and identically distributed Rician random variables, with the Rician factor of $\beta$. In addition, we use $g_{i j}=\sqrt{\varphi_{i j}} \bar{g}_{i j} \in \mathbb{C}$ to denote the CCI channel from uplink user $j$ to downlink user $i$. Let us denote the total system bandwidth $(\mathrm{Hz})$ and noise power spectral density $(\mathrm{dBm} / \mathrm{Hz})$ at receivers $(\mathrm{BS}$ and downlink users) by $B$ and $N_{0}$, respectively.

\subsection{User Grouping Method}

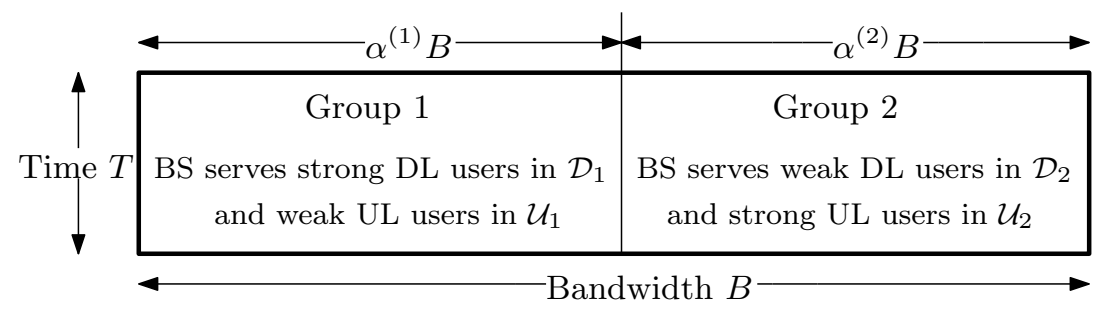

Figure 2. IBFD operation: BS simultaneously serves sets of strong downlink users in $\mathcal{D}_{1}$ and weak uplink users in $\mathcal{U}_{1}$ using a portion of system bandwidth $\alpha^{(1)} B$, and sets of weak downlink users in $\mathcal{D}_{2}$ and strong uplink users in $\mathcal{U}_{2}$ using the remaining portion of system bandwidth $\alpha^{(2)} B$.

Inspired from $[14,20,21]$, we divide all downlink users into two disjoint groups, called strong downlink and weak downlink users with respect to the distance from BS, which are denoted by $\mathcal{D}_{1}$ and $\mathcal{D}_{2}$, respectively. Similarly, all upklink users are divided into strong and weak users, denoted by $\mathcal{U}_{2}$ and $\mathcal{U}_{1}$, respectively. It is true that

$$
\left|\mathcal{D}_{1}\right|+\left|\mathcal{D}_{2}\right|=|\mathcal{D}| \text {, and }\left|\mathcal{U}_{1}\right|+\left|\mathcal{U}_{2}\right|=|\mathcal{U}| .
$$

We define Group-1 including strong downlink users $\mathcal{D}_{1}$ and weak uplink users $\mathcal{U}_{1}$, and Group-2 including weak downlink users $\mathcal{D}_{2}$ and strong uplink users $\mathcal{U}_{2}$. Let $\alpha^{(k)} \in(0,1)$ with $k \in\{1,2\}$ be fractions of system bandwidth, satisfying $\alpha^{(1)}+\alpha^{(2)}=1$. BS allocates $\alpha^{(1)} B$ bandwidth to Group-1 and the remaining bandwidth $\alpha^{(2)} B$ to Group-2, as shown in Fig. 2. By this way, strong SI and CCI are significantly mitigated since weak downlink users and uplink user are well separated. In addition, the inter-group interference is perfectly canceled. We notice that BS still operates in IBFD mode in both Group-1 and Group-2 during each communication time block $T$, which is normalize to one for simplicity. It is expected that the system performance will be greatly improved.

The UE grouping is based on the mean-square of the channel gain. To doing so, we define the following thresholds:

$$
\sigma_{\mathrm{dl}}=\frac{\sum_{i \in \mathcal{D}} \varphi_{i}^{\mathrm{d} \mathrm{l}}}{|\mathcal{D}|}
$$

and

$$
\sigma_{\mathrm{ul}}=\frac{\sum_{j \in \mathcal{U}} \varphi_{i}^{\mathrm{ul}}}{|\mathcal{U}|}
$$


which are the average of channel gains of downink users and uplink users, respectively. A dowlink user $i$ belongs to $\mathcal{D}_{1}$ if its mean-square of the channel gain is larger than or equal to $\sigma_{\mathrm{dl}}$, and vice versa. This is mathematically formulated as

$$
\mathcal{D}_{1} \triangleq\left\{\forall i \in \mathcal{D} \mid \varphi_{i}^{\mathrm{dl}}>=\sigma_{\mathrm{dl}}\right\} \text {, and } \mathcal{D}_{2} \triangleq\left\{\forall i \in \mathcal{D} \mid \varphi_{i}^{\mathrm{dl}}<\sigma_{\mathrm{dl}}\right\} .
$$

Similarly to uplink users, we have

$$
\mathcal{U}_{2} \triangleq\left\{\forall j \in \mathcal{U} \mid \varphi_{j}^{\mathrm{ul}}>=\sigma_{\mathrm{ul}}\right\}, \text { and } \mathcal{U}_{1} \triangleq\left\{\forall j \in \mathcal{U} \mid \varphi_{j}^{\mathrm{ul}}<\sigma_{\mathrm{ul}}\right\} .
$$

It is worth mentioning that at the beginning of each communication time block, CSIs of all users can be acquired at BS using a specific channel estimation scheme. Thus, the calculations in (3) and (4) are done easily.

\subsection{Beamforming Design}

Let $\mathbf{w}_{i}^{(1)} \in \mathbb{C}^{N \times 1}$ and $\mathbf{w}_{i}^{(2)} \in \mathbb{C}^{N \times 1}$ be the transmit beamforming vectors to convey independent symbols $x_{i}^{(1)}$ with $\mathbb{E}\left\{\left|x_{i}^{(1)}\right|^{2}\right\}=1$ and $x_{i}^{(2)}$ with $\mathbb{E}\left\{\left|x_{i}^{(2)}\right|^{2}\right\}=1$ to downlink users in $\mathcal{D}_{1}$ and $\mathcal{D}_{2}$, respectively. Similarly, the power coefficients of uplink users in $\mathcal{U}_{1}$ and $\mathcal{U}_{2}$ to transmit uplink symbols $\tilde{x}_{i}^{(1)}$ with $\mathbb{E}\left\{\left|\tilde{x}_{i}^{(1)}\right|^{2}\right\}=1$ and $\tilde{x}_{i}^{(2)}$ with $\mathbb{E}\left\{\left|\tilde{x}_{i}^{(2)}\right|^{2}\right\}=1$ to BS are denoted by $p_{j}^{(1)}$ and $p_{j}^{(2)}$, respectively. The signal received at downlink user $i$ in Group- $k$ with $k=\{1,2\}$ can be expressed as

$$
y_{i}^{(k)}=\mathbf{h}_{i}^{\mathrm{d} \mathrm{l},(k)} \mathbf{w}_{i}^{(k)} x_{i}^{(k)}+\sum_{i^{\prime} \in \mathcal{D}_{k} \backslash\{i\}} \mathbf{h}_{i}^{\mathrm{d} \mathrm{l},(k)} \mathbf{w}_{i^{\prime}}^{(k)} x_{i^{\prime}}^{(k)}+\sum_{j \in \mathcal{U}_{k}} \sqrt{p_{j}^{(k)}} g_{i j} \tilde{x}_{j}^{(k)}+n_{i}^{(k)}, \forall i, k=\{1,2\}
$$

where $n_{i}^{(k)} \sim \mathcal{C N}\left(0, N_{0}\right)$ is the additive white Gaussian noise (AWGN). The second and third terms in (7) are mutliuser interference and the aggregated CCI from all uplink users caused to dowlink user $i$ in the same Group- $k$, respectively. The received signals of uplink users in Group- $k$ at BS can be expressed as

$$
\mathbf{y}_{b s}^{(k)}=\sum_{j \in \mathcal{U}_{k}} \sqrt{p_{j}^{(k)}} \mathbf{h}_{j}^{\mathrm{ul},(k)} \tilde{x}_{j}^{(k)}+\sqrt{\rho} \sum_{i \in \mathcal{D}_{k}} \mathbf{H}_{\mathrm{SI}} \mathbf{w}_{i}^{(k)} x_{i}^{(k)}+\mathbf{n}_{b s}^{(k)}
$$

where $\mathbf{n}_{b s}^{(k)} \sim \mathcal{C N}\left(\mathbf{0}, N_{0} \mathbf{I}_{N}\right)$ denotes the AWGN at BS. The term $\sqrt{\rho} \sum_{i \in \mathcal{D}_{k}} \mathbf{H} \mathbf{w}_{i}^{(k)} x_{i}^{(k)}$ is the total SI caused by downlink signals to the reception of uplink at BS.

Towards a low beamforming design, we adopt ZF beamfomer/receiver at BS to cancel downlink and uplink multiuser interference, i.e., $\sum_{i^{\prime} \in \mathcal{D}_{k} \backslash\{i\}} \mathbf{h}_{i}^{\mathrm{d} \mathrm{l},(k)} \mathbf{w}_{i^{\prime}}^{(k)} x_{i^{\prime}}^{(k)} \approx 0$ and $\sum_{j^{\prime} \in \mathcal{U}_{k} \backslash\{j\}} p_{j^{\prime}}^{(k)} \mathbf{h}_{j^{\prime}}^{\mathrm{ul},(k)} \tilde{x}_{j^{\prime}}^{(k)} \approx 0$. In this paper, the total number of BS' antennas is assumed to be larger than the number of UEs, i.e., $N>\max \{D, U\}$, making ZF feasible. Let us define $\mathbf{H}^{\mathrm{dl},(k)} \triangleq\left[\left(\mathbf{h}_{1}^{\mathrm{dl},(k)}\right)^{H}, \cdots,\left(\mathbf{h}_{\left|\mathcal{D}_{k}\right|}^{\mathrm{dl},(k)}\right)^{H}\right]^{H} \in \mathbb{C}^{\left|\mathcal{D}_{k}\right| \times N}$

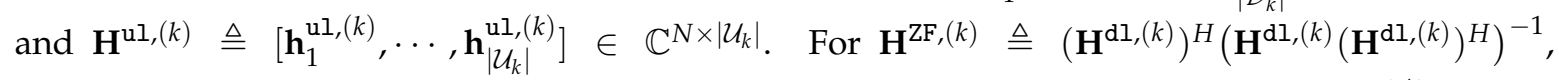
ZF beamformer for downlink users in Group- $k$ is given by $\mathbf{W}^{\mathrm{ZF},(k)}=\mathbf{H}^{\mathrm{ZF},(k)}\left(\Theta^{(k)}\right)^{1 / 2}$, where $\mathbf{\Theta}^{(k)}=\operatorname{diag}\left(\left[w_{1}^{(k)} \cdots w_{\left|\mathcal{D}_{k}^{(k)}\right|}\right]\right)$ and $w_{i}^{(k)}$ is the weight for downlink user $i$ in Group- $k$ which will be optimized later, instead of optimizing the beamforming vector with the size of $N$ variables. The signal-to-interference-plus-noise ratio (SINR) of downlink user $i$ in Group- $k$ is given by:

$$
\operatorname{SINR}_{i}^{\mathrm{dl},(k)}=\frac{w_{i}^{(k)}\left|\mathbf{h}_{i}^{\mathrm{dl},(k)} \mathbf{H}_{i}^{\mathrm{ZF},(k)}\right|^{2}}{\sum_{j \in \mathcal{U}_{k}} p_{j}^{(k)}\left|g_{i j}\right|^{2}+\alpha^{(k)} B N_{0}}
$$


where $\mathbf{H}_{i}^{\mathrm{ZF},(k)}$ is the $i$-th column of the $\mathbf{H}^{\mathrm{ZF},(k)}$. Similarly, ZF receiver for uplink users in Group- $k$ is computed as $\mathbf{A}^{\mathrm{ZF},(k)} \triangleq\left(\left(\mathbf{H}^{\mathrm{ul},(k)}\right)^{H} \mathbf{H}^{\mathrm{ul},(k)}\right)^{-1}\left(\mathbf{H}^{\mathrm{ul},(k)}\right)^{H}$. The SINR of uplink user $j$ in Group- $k$ is given by:

$$
\operatorname{SINR}_{j}^{\mathrm{ul},(k)}=\frac{p_{j}^{(k)}\left|\mathbf{A}_{j}^{\mathrm{ZF},(k)} \mathbf{h}_{j}^{\mathrm{ul},(k)}\right|^{2}}{\rho\left\|\mathbf{A}_{j}^{\mathrm{ZF},(k)} \mathbf{H}_{\mathrm{SI}} \mathbf{W}^{\mathrm{ZF},(k)}\right\|^{2}+\alpha^{(k)} B N_{0}\left\|\mathbf{A}_{j}^{\mathrm{ZF},(k)}\right\|^{2}}
$$

where $\mathbf{A}_{j}^{\mathrm{ZF},(k)}$ is the $j$-th row of the $\mathbf{A}^{\mathrm{ZF},(k)}$.

\subsection{Optimization Problem Formulation}

From (9) and (10), the data rate in nats/s of downlink user $i$ and uplink user $j$ in Group- $k$ are given as

$$
R_{i}^{\mathrm{dl},(k)}\left(w_{i}^{(k)},\left\{p_{j}^{(k)}\right\}, \alpha^{(k)}\right)=\alpha^{(k)} B \ln \left(1+\operatorname{SINR}_{i}^{\mathrm{dl},(k)}\right)
$$

and

$$
R_{j}^{\mathrm{ul},(k)}\left(\left\{w_{i}^{(k)}\right\}, p_{j}^{(k)}, \alpha^{(k)}\right)=\alpha^{(k)} B \ln \left(1+\operatorname{SINR}_{j}^{\mathrm{ul},(k)}\right)
$$

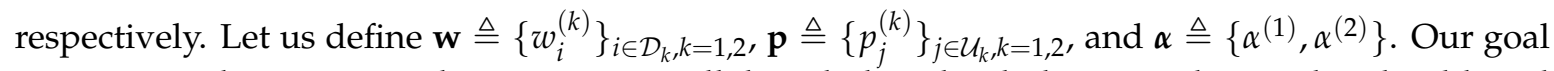
is maximize the minimum data rate among all downlink and uplink users subject to bandwidth and power constraint, which is stated as:

$$
\begin{aligned}
\max _{\mathbf{w}, \mathbf{p}, \boldsymbol{\alpha}} & \min _{i \in \mathcal{D}_{k}, j \in \mathcal{U}_{k}, k=\{1,2\}}\left\{R_{i}^{\mathrm{dl},(k)}\left(w_{i}^{(k)},\left\{p_{j}^{(k)}\right\}, \alpha^{(k)}\right), R_{j}^{\mathrm{ul},(k)}\left(\left\{w_{i}^{(k)}\right\}, p_{j}^{(k)}, \alpha^{(k)}\right)\right\} \\
\text { s.t. } & \sum_{k=1}^{2} \operatorname{tr}\left(\left(\mathbf{H}^{\mathrm{ZF},(k)}\right)^{H} \mathbf{H}^{\mathrm{ZF},(k)} \mathbf{\Theta}^{(k)}\right) \leq P_{b s}^{\max } \\
& p_{j}^{(k)} \leq P_{j}^{(k), \max }, \forall j \in \mathcal{U}_{k}, k=\{1,2\} \\
& \sum_{k=1}^{2} \alpha^{(k)} \leq 1
\end{aligned}
$$

where (13b) and (13c) are power constraints at BS and uplink user $j$ in Group- $k$, respectively.

\section{Proposed Iterative Algorithm}

In problem (13), all constraints are linear but the objective function (13a) is non-concave and non-smooth. As a consequence, a direct application of IA method [22,23] is not applicable. Several transformations are required to convert (13) into a more tractable form. To doing so, we first introduce the following theorem to facilitate design.

Theorem 1. Problem (13) is equivalently rewritten as:

$$
\begin{array}{rl}
\max _{\mathbf{w}, \mathbf{p}, \alpha, \gamma, r} & r \\
\text { s.t. } & \alpha^{(k)} B \ln \left(1+\gamma_{i}^{\mathrm{dl},(k)}\right) \geq r, \forall i \in \mathcal{D}_{k}, k=\{1,2\} \\
& \alpha^{(k)} B \ln \left(1+\gamma_{j}^{\mathrm{ul},(k)}\right) \geq r, \forall j \in \mathcal{U}_{k}, k=\{1,2\} \\
& \operatorname{SINR}_{i}^{\mathrm{dl},(k)} \geq \gamma_{i}^{\mathrm{dl},(k)}, \forall i \in \mathcal{D}_{k}, k=\{1,2\} \\
& \operatorname{SINR}_{j}^{\mathrm{ul},(k)} \geq \gamma_{j}^{\mathrm{ul},(k)}, \forall j \in \mathcal{U}_{k}, k=\{1,2\} \\
& (13 \mathrm{~b}),(13 \mathrm{c}),(13 \mathrm{~d})
\end{array}
$$

where $\gamma=\left\{\gamma_{i}^{\mathrm{dl},(k)}, \gamma_{j}^{\mathrm{ul},(k)}\right\}_{i, j, k}$ and $r$ are new variables. 
Proof. The equivalence between (13) and (14) is attributed to that fact that all constraints (14b)-(14e) must hold with equality at the optimum, which can be verified by contradiction. Suppose that constraints (14d) and (14e) do not hold with equality at the optimum, it will result in larger value of left-hand sides of (14b) and (14c). In other words, a strictly large objective value can be obtained, which contradicts with the assumption of optimum.

We can see that the objective is a linear function, while constraints (14b)-(14e) are nonconvex. We now apply IA method to tackle nonconvex part of problem (14). The following lemma is introduced to approximate constraints (14b) and (14c).

Lemma 1. We first rewrite (14b) and (14c) as

$$
\begin{aligned}
\alpha^{(k)} \ln \left(1+\gamma_{i}^{\mathrm{dl},(k)}\right) & \geq \frac{r}{B}, \forall i \in \mathcal{D}_{k}, k=\{1,2\} \\
\alpha^{(k)} B \ln \left(1+\gamma_{j}^{\mathrm{ul},(k)}\right) & \geq \frac{r}{B}, \forall j \in \mathcal{U}_{k}, k=\{1,2\} .
\end{aligned}
$$

Let $\left(\bar{\alpha}^{(k)}, \bar{\gamma}_{i}^{\mathrm{dl},(k)}, \bar{\gamma}_{j}^{\mathrm{ul},(k)}\right)$ be feasible points of $\left(\alpha^{(k)}, \gamma_{i}^{\mathrm{dl},(k)}, \gamma_{j}^{\mathrm{ul},(k)}\right)$ at iteration $\kappa$ (which will be updated after each iteration). The nonconvex constraints (14b) and (14c) are approximated as

$$
\begin{gathered}
2 \bar{\alpha}^{(k)} \ln \left(1+\bar{\gamma}_{i}^{\mathrm{dl},(k)}\right)+\frac{\bar{\alpha}^{(k)} \bar{\gamma}_{i}^{\mathrm{dl},(k)}}{\bar{\gamma}_{i}^{\mathrm{dl},(k)}+1}-\frac{\bar{\alpha}^{(k)}\left(\bar{\gamma}_{i}^{\mathrm{dl},(k)}\right)^{2}}{\bar{\gamma}_{i}^{\mathrm{dl},(k)}+1} \frac{1}{\gamma_{i}^{\mathrm{dl},(k)}} \\
-\left(\bar{\alpha}^{(k)}\right)^{2} \ln \left(1+\bar{\gamma}_{i}^{\mathrm{dl},(k)}\right) \frac{1}{\alpha^{(k)}} \geq \frac{r}{B}, \forall i \in \mathcal{D}_{k}, k=\{1,2\} \\
2 \bar{\alpha}^{(k)} \ln \left(1+\bar{\gamma}_{j}^{\mathrm{ul},(k)}\right)+\frac{\bar{\alpha}^{(k)} \bar{\gamma}_{j}^{\mathrm{ul},(k)}}{\bar{\gamma}_{j}^{\mathrm{ul},(k)}+1}-\frac{\bar{\alpha}^{(k)}\left(\bar{\gamma}_{j}^{\mathrm{ul},(k)}\right)^{2}}{\bar{\gamma}_{j}^{\mathrm{ul},(k)}+1} \frac{1}{\gamma_{j}^{\mathrm{ul},(k)}} \\
-\left(\bar{\alpha}^{(k)}\right)^{2} \ln \left(1+\bar{\gamma}_{j}^{\mathrm{ul},(k)}\right) \frac{1}{\alpha^{(k)}} \geq \frac{r}{B}, \forall j \in \mathcal{U}_{k}, k=\{1,2\}
\end{gathered}
$$

which are convex constraints.

Proof. The proof is based on [21], which is detailed in Appendix A

Next, we address nonconvex constraints (14d) and (14e), which can be re-expressed as

$$
\begin{aligned}
& \left|\mathbf{h}_{i}^{\mathrm{dl},(k)} \mathbf{H}_{i}^{\mathrm{ZF},(k)}\right|^{2} \frac{w_{i}^{(k)}}{\gamma_{i}^{\mathrm{dl},(k)}} \geq \sum_{j \in \mathcal{U}_{k}} p_{j}^{(k)}\left|g_{i j}\right|^{2}+\alpha^{(k)} B N_{0}, \forall i \in \mathcal{D}_{k}, k=\{1,2\} \\
& \left|\mathbf{A}_{j}^{\mathrm{ZF},(k)} \mathbf{h}_{j}^{\mathrm{ul},(k)}\right|^{2} \frac{p_{j}^{(k)}}{\gamma_{j}^{\mathrm{ul},(k)}} \geq \rho\left\|\mathbf{A}_{j}^{\mathrm{ZF},(k)} \mathbf{H}_{\mathrm{SI}} \mathbf{W}^{\mathrm{ZF},(k)}\right\|^{2}+\alpha^{(k)} B N_{0}\left\|\mathbf{A}_{j}^{\mathrm{ZF},(k)}\right\|^{2}, \forall j \in \mathcal{U}_{k}, k=\{1,2\} .
\end{aligned}
$$

Both right-hand sides of (17a) and (17b) are linear functions. In (17a), we can rewrite $\frac{w_{i}^{(k)}}{\gamma_{i}^{\mathrm{d} l(k)}}$ as $\frac{\left(\sqrt{w_{i}^{(k)}}\right)^{2}}{\gamma_{i}^{\mathrm{d},(k)}}$ which is convex in $\left(\sqrt{w_{i}^{(k)}}, \gamma_{i}^{\mathrm{dl},(k)}\right)$. As a result, (17a) is innerly approximated as

$$
\begin{aligned}
& \left|\mathbf{h}_{i}^{\mathrm{dl},(k)} \mathbf{H}_{i}^{\mathrm{ZF},(k)}\right|^{2}\left(2 \frac{\sqrt{\bar{w}_{i}^{(k)}}}{\bar{\gamma}_{i}^{\mathrm{dl},(k)}} \sqrt{w_{i}^{(k)}}-\frac{\bar{w}_{i}^{(k)}}{\left(\bar{\gamma}_{i}^{\mathrm{dl},(k)}\right)^{2}} \gamma_{i}^{\mathrm{dl},(k)}\right) \\
& \geq \sum_{j \in \mathcal{U}_{k}} p_{j}^{(k)}\left|g_{i j}\right|^{2}+\alpha^{(k)} B N_{0}, \forall i \in \mathcal{D}_{k}, k=\{1,2\} .
\end{aligned}
$$




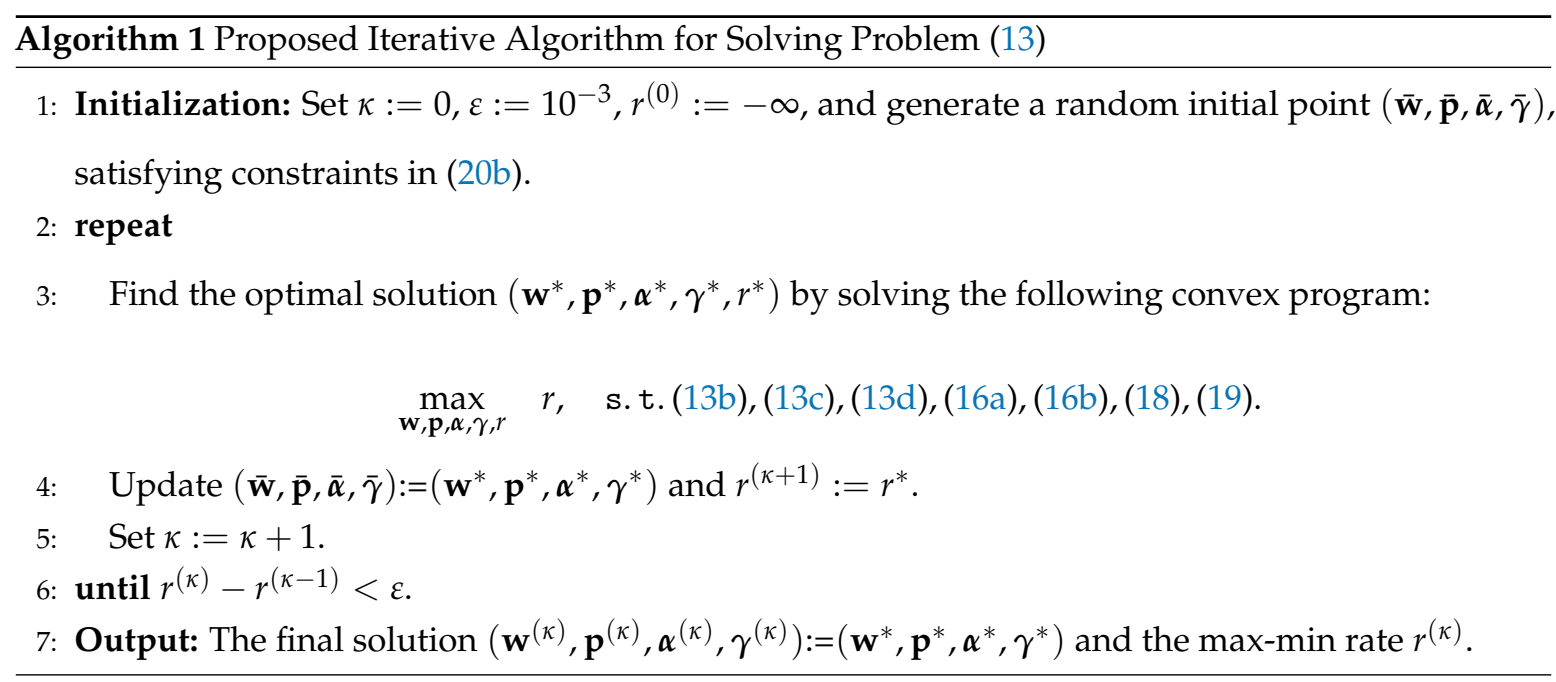

Table 1. Simulation Parameters

\begin{tabular}{l|l}
\hline Simulation Parameter & Value \\
\hline \hline System bandwidth & $10 \mathrm{MHz}$ \\
Noise power spectral density at BS and uplink users & $N_{0}=-174 \mathrm{dBm} / \mathrm{Hz}$ \\
Path loss between BS and users & $103.8+20.9 \log _{10}(d) \mathrm{dB}$ \\
Path loss between a uplink user and a downlink user & $145.4+37.5 \log _{10}(d) \mathrm{dB}$ \\
Power budget at BS & $P_{b s}^{\max }=26 \mathrm{dBm}$ \\
Power budget at uplink users & $P_{j}^{(k), \max }=23 \mathrm{dBm}, \forall j, k$ \\
Level of residual SI, $\rho$ & $-75 \mathrm{~dB}$ \\
Number of antennas at BS & $N=5$ \\
Number of downlink users & $D=4$ \\
Number of uplink users & $U=4$ \\
\hline
\end{tabular}

Similarly for (17b), we have

$$
\begin{aligned}
& \left|\mathbf{A}_{j}^{\mathrm{ZF},(k)} \mathbf{h}_{j}^{\mathrm{ul},(k)}\right|^{2}\left(2 \frac{\sqrt{\bar{p}_{j}^{(k)}}}{\bar{\gamma}_{j}^{\mathrm{ul},(k)}} \sqrt{p_{j}^{(k)}}-\frac{\bar{p}_{j}^{(k)}}{\left(\bar{\gamma}_{j}^{\mathrm{ul},(k)}\right)^{2}} \gamma_{j}^{\mathrm{ul},(k)}\right) \\
& \geq \rho\left\|\mathbf{A}_{j}^{\mathrm{ZF},(k)} \mathbf{H}_{\mathrm{SI}} \mathbf{W}^{\mathrm{ZF},(k)}\right\|^{2}+\alpha^{(k)} B N_{0}\left\|\mathbf{A}_{j}^{\mathrm{ZF},(k)}\right\|^{2}, \forall j \in \mathcal{U}_{k}, k=\{1,2\} .
\end{aligned}
$$

In summary, the convex approximate problem of (13) solved at iteration $\kappa$ is given as

$$
\begin{array}{r}
\max _{\mathbf{w}, \mathbf{p}, \boldsymbol{\alpha}, \gamma, r} r \\
\text { s.t. }(13 \mathrm{~b}),(13 \mathrm{c}),(13 \mathrm{~d}),(16 \mathrm{a}),(16 \mathrm{~b}),(18),(19) .
\end{array}
$$

The proposed iterative algorithm to solve (13) is summarized in Algorithm 1, where $\varepsilon>0$ is a very small constant. Following the proof of convergence of IA-based iterative algorithm in [23], Algorithm 1 will converge to at least a local optimal solution to problem (13).

Computational complexity analysis: The major complexity of Algorithm 1 is due to solving problem (20) in Step 3, which involves $2 D+2 U+3$ optimization variables and $3 U+2 D+2$ quadratic and linear constraints. As a result, the per-iteration computational complexity of Algorithm 1 is $\mathcal{O}((3 U+$ $\left.2 D+2)^{2.5}\left((2 D+2 U+3)^{2}+3 U+2 D+2\right)\right)$, following [24]. 


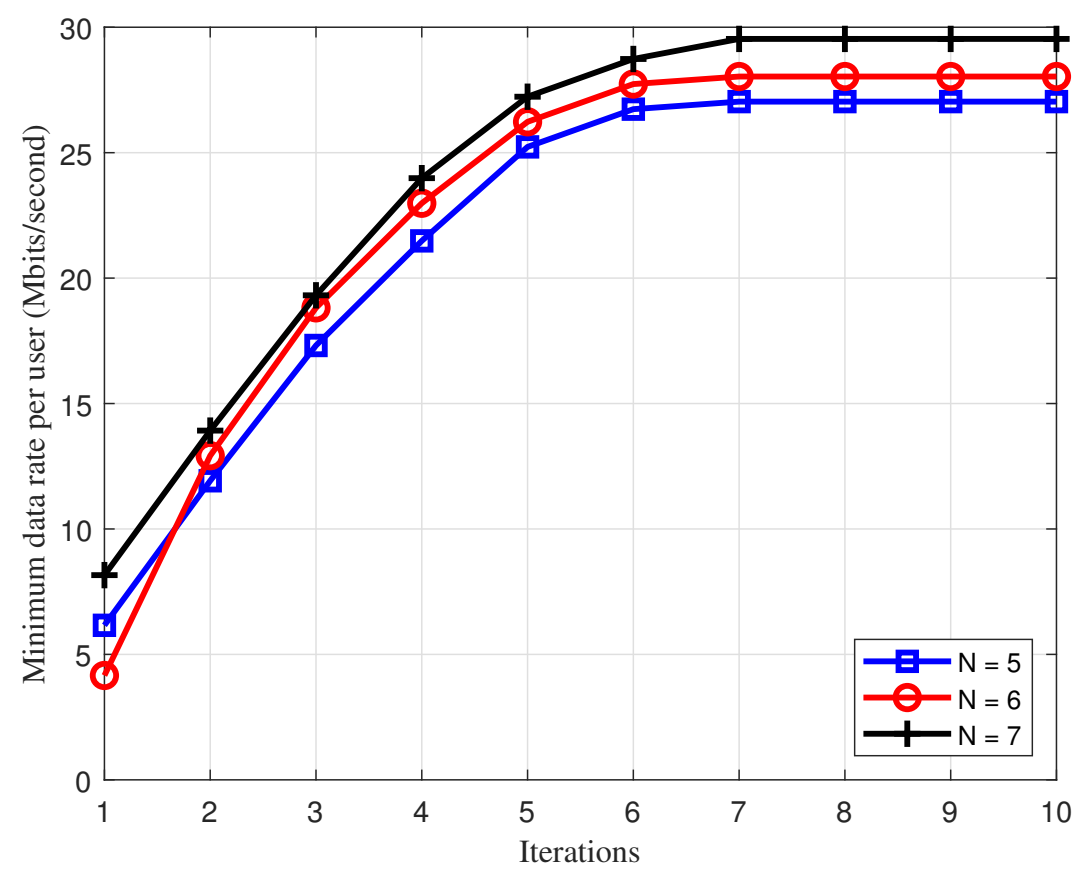

Figure 3. Convergence behavior of Algorithm 1 with the difference number of antennas at BS.

\section{Numerical Results and Discussions}

In this section, we evaluate the performance of Algorithm 1 using computer simulation. All downlink and uplink users are randomly distributed in the area of a circle with radius of $100 \mathrm{~m}$. Unless stated otherwise, the main simulation parameters are given in Table 1, following studies in $[7,10,21]$. We set the same power budget for all uplink users. The SI channel matrix $\mathbf{H}_{\mathrm{SI}}$ is generated as independent and identically distributed Rician random variables with Rician factor $5 \mathrm{~dB}$. In Table 1 , parameter $d$ is the distance between a transmitter and a receiver. In Step 6 of Algorithm 1, we set $\varepsilon=10^{-3}$. We use the convex solver SeDuMi to solve the convex program in the MATLAB environment. In the following figures, the results are averaged over 1000 simulation trials.

To demonstrate the effectiveness of the proposed algorithm, we also consider the following existing schemes:

- "Conventional FD:" In this scheme, all downlink and uplink users are served in the same time-frequency resource (without user groping) [12]).

- "Algorithm 1 with $\alpha^{(k)}=0.5, \forall k:$ " The solution of this scheme can be easily obtained with a slight modification of Algorithm 1.

- "Half-duplex:" BS servers all downlink and uplink users in the same frequency resource but in two separate time blocks.

In Fig. 3, we explore the convergent property of the proposed Algorithm 1. First, we can see that the minimum data rate is monotonically increased when the number of iterations increases, which is aligned with the IA principles. Second, Algorithm 1 takes about seven iterations to converge to the optimal solution. The convergence pattern is quite independent with the problem size (i.e., the number of BS's antennas N). The reason for this is that the convex problem (20) does not involve with $N$, which further confirms the low complexity of the proposed algorithm. Of course, increasing the number of BS's antennas will improve the minimum data rate per user since more degrees-of-freedom are added to the system to support multiple users and reduce interference.

We show the effect of the level residual SI on the average minimum data rate, $\rho \in[-110-30]$ $\mathrm{dB}$, in Fig. 4. As can be seen, the performance of all FD schemes is degraded when $\rho$ increases. It is 


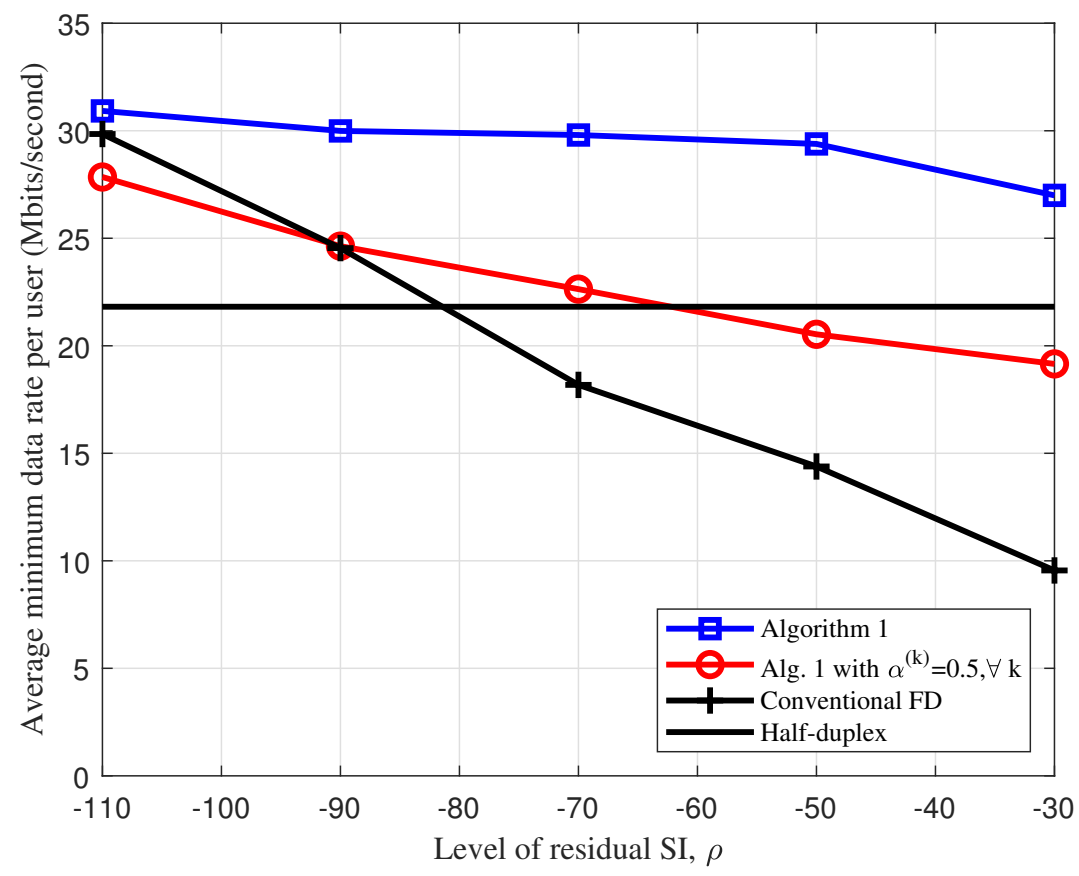

Figure 4. Effect of the level residual SI on the average minimum data rate, $\rho[\mathrm{dB}]$.

clear that the higher the level residual SI the stronger the SI power caused at BS. Unsurprisingly, the performance of half-duplex scheme is independent with $\rho$ since downlink and uplink are operated at two separate time blocks, and thus there is no SI at BS. Notably, Algorithm 1 provides the best performance among all considered schemes in all range of $\rho$. The minimum data rate of Algorithm 1 is mostly unchanged in typical range of $\rho \in[-110-50] \mathrm{dB}$ and is slightly degraded when $\rho>-50 \mathrm{~dB}$. These observations further confirm the effectiveness of the proposed algorithm with user grouping and jointly optimizing all variables to handle the whole network interference more effectively.

Finally, we plot the average data rate versus the transmit power at BS, $P_{b s}^{\max } \in[1430] \mathrm{dBm}$. The performance of half-duplex is unchanged since the minimum data rate is mainly determined by users in uplink. We can see that the performance of FD-based schemes is significantly improved when $P_{b s}^{\max }$ from $14 \mathrm{dBm}$ to $22 \mathrm{dBm}$, and then sightly increased when $P_{b s}^{\max }$ is large. This is because BS with higher power budget $P_{b s}^{\max }$ will cause stronger SI to the reception of upink users. To maintain the max-min data rate among all users, BS has to scale down its transmit power to reduce the effect of SI power. Again, Algorithm 1 sill offers the best performance among all FD schemes and haft-duplex scheme in the practical value of $P_{b s}^{\max }=26 \mathrm{dBm}$.

\section{Conclusion}

In this paper, we considered the max-min rate fairness optimization for IBFD IoT networks. We proposed a simple yet efficient user grouping method, aiming for an efficient network interference management. An ZF-based design was adopted in both downlink and uplink to reduce the computational complexity of the problem design. The formulated problem has a highly non-concave objective function subject to linear constraint. Towards a low-complexity solution, we first converted the original problem to a nonconvex problem but with more tractable form. We then developed an a low-complexity iterative algorithm based on IA framework that converges to at least a local optimum. Numerical results were provided to demonstrate the significant performance improvement of the proposed method compared to existing ones.

Conflicts of Interest: The author declares no conflict of interest. 


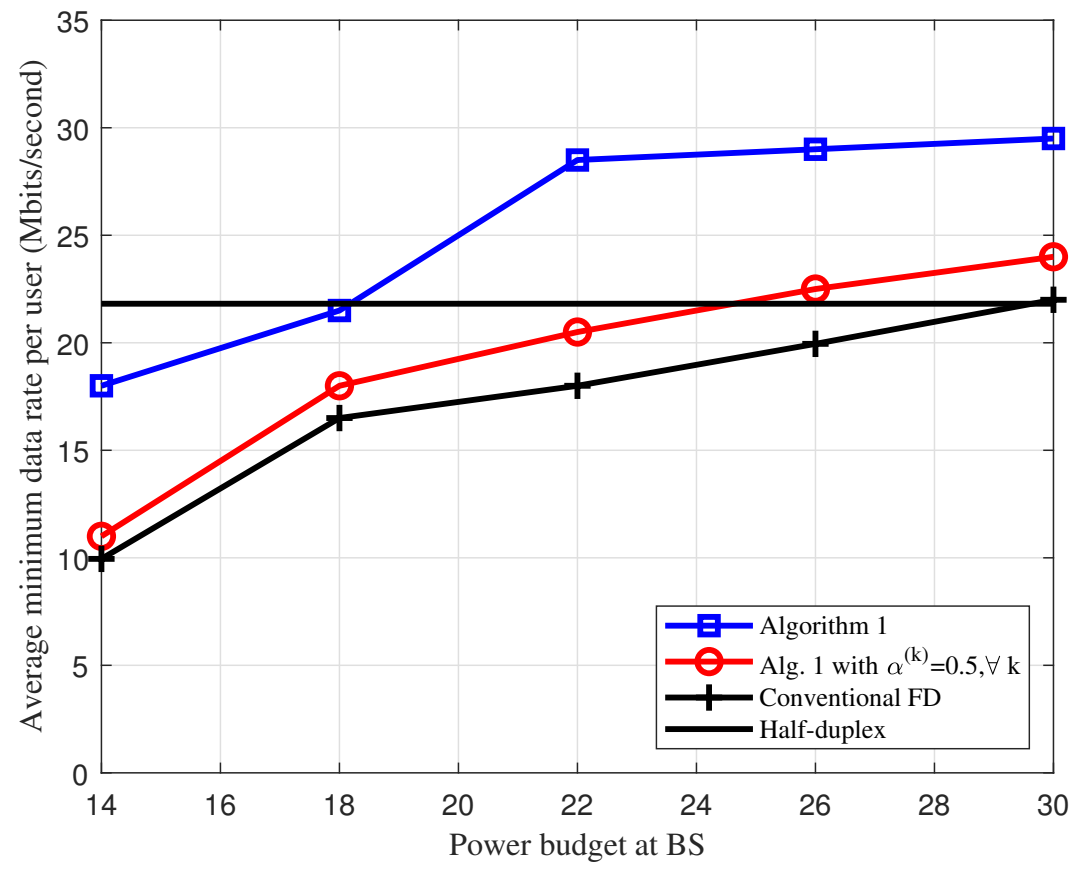

Figure 5. Average minimum data rate versus the transmit power at BS, $P_{b s}^{\max }[\mathrm{dBm}]$.
Abbreviations
The following abbreviations are used in this manuscript:
IBFD In-band full-duplex
HD Half-duplex
IA Inner approximation
BS Base station
SI Self-interference
CCI Co-channel interference
CSI Channel state information
5G Fifth-generation wireless network
IoT Internet of Things
SEM Spectral efficiency maximization
DoF Degrees-of-freedom
ZF Zero-forcing
NOMA Non-orthogonal multiple access
DL Downlink
UL Uplink 


\section{Appendix A Proof of Lemma 1}

We first consider the function $\ln (1+1 / x) / y$, which is convex in the domain $x, y \in \mathbb{R}_{+}$. The first order Taylor approximation of $\ln (1+1 / x) / y$ is given as:

$$
\begin{aligned}
\frac{\ln \left(1+\frac{1}{x}\right)}{y} \geq & \frac{\ln \left(1+\frac{1}{\bar{x}}\right)}{\bar{y}}-\left.\nabla_{x} \frac{\ln \left(1+\frac{1}{x}\right)}{\bar{y}}\right|_{\bar{x}}(x-\bar{x}), \\
& \left.\nabla_{y} \frac{\ln \left(1+\frac{1}{\bar{x}}\right)}{y}\right|_{\bar{y}}(y-\bar{y}) \\
= & 2 \frac{\ln \left(1+\frac{1}{\bar{x}}\right)}{\bar{y}}+\frac{1}{\bar{y}(\bar{x}+1)} \\
& -\frac{1}{\bar{y} \bar{x}(\bar{x}+1)} x-\frac{\ln \left(1+\frac{1}{\bar{x}}\right)}{\bar{y}^{2}} y .
\end{aligned}
$$

The results in (16) are obtained by setting: $x^{-1}=\gamma_{i}^{\mathrm{dl},(k)}, x^{-1}=\gamma_{j}^{\mathrm{ul},(k)}$ and $y^{-1}=\alpha^{(k)}$.

\section{References}

1. Palattella, M.R.; Dohler, M.; Grieco, A.; Rizzo, G.; Torsner, J.; Engel, T.; Ladid, L. Internet of Things in the 5G Era: Enablers, Architecture, and Business Models. IEEE J. Sel. Areas Commun. 2016, 34, 510-527. doi:10.1109/JSAC.2016.2525418.

2. Cisco Visual Networking Index: Global Mobile Data Traffic Forecast Update, 2016-2021, 2017, pp. [Online] https://www.cisco.com/c/en/us/solutions/collateral/serviceprovider/ visual-networking-index-vni/mobile-white-paper-c11-520862.html.

3. Ericsson Mobility Report. Ericsson AB, Stockholm, Sweden 2018, p. [Online] https://goo.gl/bSxgss.

4. Andrews, J.; Buzzi, S.; Choi, W.; Hanly, S.; Lozano, A.; Soong, A.; Zhang, J. What Will 5G Be? IEEE J. Select. Area Commun. 2014, 32, 1065-1082. doi:10.1109/JSAC.2014.2328098.

5. Zhang, Z.; Long, K.; Vasilakos, A.V.; Hanzo, L. Full-Duplex Wireless Communications: Challenges, Solutions, and Future Research Directions. Proceedings of the IEEE 2016, 104, 1369-1409. doi:10.1109/JPROC.2015.2497203.

6. Zhang, Z.; Chai, X.; Long, K.; Vasilakos, A.V.; Hanzo, L. Full duplex techniques for 5 G networks: Self-interference cancellation, protocol design, and relay selection. IEEE Commun. Mag. 2015, 53, 128-137. doi:10.1109/MCOM.2015.7105651.

7. Bharadia, D.; McMilin, E.; Katti, S. Full duplex radios. Proc. ACM SIGCOMM Computer Commun. Review, 2013, Vol. 43, pp. 375-386.

8. Riihonen, T.; Werner, S.; Wichman, R. Mitigation of loopback self-interference in full-duplex MIMO relays. IEEE Trans. Signal Process. 2011, 59, 5983-5993.

9. System scenarios and technical requirements for full-duplex concept. DUPLO project, Deliverable D1.1.

10. Duarte, M.; Dick, C.; Sabharwal, A. Experiment-driven characterization of full-duplex wireless systems. IEEE Trans. Wireless Commun. 2012, 11, 4296-4307.

11. Sabharwal, A.; Schniter, P.; Guo, D.; Bliss, D.W.; Rangarajan, S.; Wichman, R. In-band full-duplex wireless: Challenges and opportunities. IEEE J. Select. Areas Commun. 2014, 32, 1637-1652.

12. Nguyen, D.; Tran, L.N.; Pirinen, P.; Latva-aho, M. On the spectral efficiency of full-duplex small cell wireless systems. IEEE Trans. Wireless Commun. 2014, 13, 4896-4910.

13. Huberman, S.; Le-Ngoc, T. MIMO Full-Duplex Precoding: A Joint Beamforming and Self-Interference Cancellation Structure. IEEE Trans. Wireless Commun. 2015, 14, 2205-2217. doi:10.1109/TWC.2014.2382589.

14. Nguyen, V.D.; Nguyen, H.V.; Nguyen, C.T.; Shin, O.S. Spectral Efficiency of Full-Duplex Multi-user System: Beamforming Design, User Grouping, and Time Allocation. IEEE Access 2017, 5, 5785-5797. doi:10.1109/ACCESS.2017.2668384. 
15. Tam, H.H.M.; Tuan, H.D.; Ngo, D.T. Successive Convex Quadratic Programming for Quality-of-Service Management in Full-Duplex MU-MIMO Multicell Networks. IEEE Trans. Commun. 2016, 64, 2340-2353. doi:10.1109/TCOMM.2016.2550440.

16. Nguyen, H.V.; Nguyen, V.D.; Dobre, O.A.; Wu, Y.; Shin, O.S. Joint antenna array mode selection and user assignment for full-duplex MU-MISO systems. IEEE Trans. Wireless Commun. 2019, 18, 2946-2963. doi:10.1109/TWC.2019.2907489.

17. Chalise, B.K.; Suraweera, H.A.; Zheng, G.; Karagiannidis, G.K. Beamforming Optimization for Full-Duplex Wireless-Powered MIMO Systems. IEEE Trans. Commun. 2017, 65, 3750-3764. doi:10.1109/TCOMM.2017.2710196.

18. Nguyen, V.D.; Duong, T.Q.; Tuan, H.D.; Shin, O.S.; Poor, H.V. Spectral and Energy Efficiencies in Full-Duplex Wireless Information and Power Transfer. IEEE Trans. Commun. 2017, 65, 2220-2233. doi:10.1109/TCOMM.2017.2665488.

19. Aquilina, P.; Cirik, A.C.; Ratnarajah, T. Weighted Sum Rate Maximization in Full-Duplex Multi-User Multi-Cell MIMO Networks. IEEE Trans. Commun. 2017, 65, 1590-1608. doi:10.1109/TCOMM.2017.2652475.

20. Nguyen, H.V.; Nguyen, V.D.; Dobre, O.A.; Nguyen, D.N.; Dutkiewicz, E.; Shin, O.S. Joint Power Control and User Association for NOMA-Based Full-Duplex Systems. IEEE Trans. Commun. 2019, 67, 8037-8055. doi:10.1109/TCOMM.2019.2933217.

21. Nguyen, V.D.; Nguyen, H.V.; Dobre, O.A.; Shin, O.S. A New Design Paradigm for Secure Full-Duplex Multiuser Systems. IEEE J. Select. Areas Commun. 2018, 36, 1480-1498. doi:10.1109/JSAC.2018.2824379.

22. Marks, B.R.; Wright, G.P. A general inner approximation algorithm for nonconvex mathematical programs. Operations Research 1978, 26, 681-683.

23. Beck, A.; Ben-Tal, A.; Tetruashvili, L. A sequential parametric convex approximation method with applications to nonconvex truss topology design problems. J. Global Optim. 2010, 47, $29-51$.

24. Peaucelle, D.; Henrion, D.; Labit, Y. Users Guide for SeDuMi Interface 1.032002. 